\title{
Biogenesis of Mitochondrial Membranes in Neurospora crassa during Cellular Differentiation: Ultrastructural Changes Accompanying Differentiation
}

\author{
By D. P. BECK* AND J. W. GREENAWALT $\dagger$ \\ Department of Physiological Chemistry, Johns Hopkins University \\ School of Medicine, Baltimore, Maryland 21205 , U.S.A.
}

(Received 5 May 1975; revised 23 July 1975)

\begin{abstract}
SUMMARY
The ultrastructural characteristics of Neurospora cells during dedifferentiation and redifferentiation of conidiospores into vegetative cells have been determined. This germination process occurs between 2 and $5 \mathrm{~h}$ after inoculation; by $3.5 \mathrm{~h}$, approximately $50 \%$ of the cells have germinated. The cells enter the exponential phase of dry-weight gain between 4 and $5 \mathrm{~h}$ after inoculation. Several unusual structures are observed in Neurospora cells during germination. Whorled structures are frequently seen in the cytoplasm during germination, and occasionally at other times. They appear to be derived from the cytoplasmic membrane. Whorled structures of different appearance were observed in the mitochondria between 2 and $4 \mathrm{~h}$ after inoculation. Their number was related to the level of metabolizable carbohydrate, and was higher in $15 \%$ glucose- than in $2 \%$ sucrosesupplemented medium, and very low in medium containing $15 \%$ mannitol, or $2 \%$ sucrose $+13 \%$ 2-deoxyglucose, or no added carbohydrate. The mitochondrial inclusions were osmiophilic and could be removed by treatment with $90 \%$ aqueous acetone in the cold, indicating that they were composed at least in part of lipid. The strong dependence of the number of mitochondrial inclusions on time and on carbohydrate supplementation, suggests that there is a physiological basis for these structures and that they reflect changes occurring in the mitochondria at times significant to cellular differentiation.
\end{abstract}

\section{INTRODUCTION}

The differentiation of relatively dormant conidiospores of Neurospora crassa into vigorously growing vegetative hyphae involves biochemical, cytological and morphogenetic changes. We have considered the changes accompanying germination and growth of $N$. crassa conidiospores as processes of differentiation as outlined by Turian (1966). Differentiation in ascomycetes is defined as any developmental change in form towards either greater complexity or simplification. Thus the already differentiated, dormant (or latent) conidiospore undergoes dedifferentiation, which is recognized morphogenetically by germ tube formation and physiologically by proliferative capacity. This stage is followed by redifferentiation into the growing vegetative hyphal cells. The dedifferentiation and redifferentiation processes are fairly well synchronized and separated in time in Neurospora. The onset of these stages can be monitored readily by following the emergence of germ tubes and by increases in dry mass, respectively.

* Present address: Maryland Psychiatric Research Centre, Baltimore, Maryland 21228, U.S.A.

† Present address: Department of Biochemistry, University of Tennessee, Knoxville, Tennessee 37916, U.S.A. 
Neurospora is a strict aerobe, in that both germination of conidiospores and vegetative growth are oxygen-dependent processes. However, although conidia do not germinate under anaerobic conditions, active fermentative metabolism indicated by glucose utilization and the formation of ethanol plus $\mathrm{CO}_{2}$ does occur in the ungerminated spores of Neurospora sitophila (Cochrane, 1966). Early studies on enzyme activity and cell differentiation in Neurospora indicated that the activity of succinic dehydrogenase, localized in or tightly bound to the inner mitochondrial membrane, is very low in spores but increases during extrusion and growth of germ tubes (Zalokar, 1959). These facts suggested that changes in the biochemical activities of mitochondria might be linked to the differentiation of conidia into vegetative cells. Thus, under appropriate growth conditions, a culture of Neurospora will contain cells at similar stages of differentiation and would appear to lend itself to a study of the biogenesis of mitochondrial function.

Hall \& Greenawalt (1964, 1967) described the means of isolating, from $N$. crassa, mitochondria which are capable of carrying out coupled oxidative phosphorylation. Results of those studies indicated that increases in the respiration of a variety of substrates and in coupled phosphorylation accompany the conversion of conidia into vegetative cells. However, only mitochondria isolated from conidia, germinating conidia and I- to 2-day-old hyphae were compared. Therefore, the kinetics of the changes observed were not ascertained and changes could not be related to specific stages of differentiation. In a study during the redifferentiation stage alone, Weiss (1965) showed that cytochrome oxidase activity is increased during exponential growth. Stine (1967, 1969) also reported that succinic dehydrogenase activity is not essential for sporulation or germination but is needed for vegetative growth.

Ultrastructural studies of Neurospora cells examined during dedifferentiation and redifferentiation of conidiospores into vegetative cells revealed (i) that in contrast to yeast cells repressed by glucose or anaerobiosis, mitochondrial ultrastructure in Neurospora conidia is essentially identical to that of mitochondria in vegetative cells, and (ii) that membrane-like inclusions are present within the mitochondria of Neurospora at early stages of growth (Greenawalt, 1965; Beck \& Greenawalt, 1968). These ultrastructural changes might reflect alterations in mitochondrial function and/or composition which occur as aerobic metabolism is induced during differentiation.

The present paper describes the major ultrastructural features which accompany dedifferentiation and redifferentiation of Neurospora spores into vegetative cells, and quantitates the appearance of mitochondrial and cytoplasmic inclusions during the process. The accompanying paper (Beck \& Greenawalt, 1976) shows that the formation of the membranous inclusions in mitochondria is correlated with increases in the rate of synthesis of mitochondrial phospholipids, and that ultrastructural and compositional changes precede development of full capacity for oxidative phosphorylation.

\section{METHODS}

Organism and growth. Neurospora crassa SY7A was obtained from the Fungal Genetics Stock Center, Humboldt State College, Ariata, California, U.S.A. Stock suspensions of conidia were prepared as described by Hall \& Greenawalt (1967) and contained about $10^{8}$ spores $/ \mathrm{ml}$. Conidia were germinated at $30^{\circ} \mathrm{C}$ in $500 \mathrm{ml}$ of Vogel's (I956) complete medium in a 21 flask. Vogel's medium was supplemented with various sugars as indicated in the text. The flasks were shaken on a New Brunswick G-Io gyrotary shaker operated at $250 \mathrm{rev} . / \mathrm{min}$. The final spore density in all media was about $10^{7} \mathrm{spores} / \mathrm{ml}$. Growth 
was measured gravimetrically, and spore germination was determined by phase-contrast microscopy.

Electron microscopy. Samples of $N$. crassa were taken at different times during germination and growth, and prepared for quantitative electron microscopy by fixation in $3 \%$ (v/v) glutaraldehyde (Sabatini, Bensch \& Barnett, 1963) followed by post-fixation in $2 \%(\mathrm{v} / \mathrm{v})$ osmium tetroxide (Millonig, 196I, 1962). The samples were then dehydrated in a graded alcohol series and embedded in Epon 812 (Luft, 196I). Thin sections, cut with a diamond knife, were stained with uranyl acetate and lead citrate (Reynolds, 1963) and observed in a Siemens Elmiskop I operated at $80 \mathrm{kV}$. The numbers of mitochondria, mitochondrial inclusions and cytoplasmic membrane whorls in these sections were counted. A minimum of 30 cells, representing between 300 and 400 mitochondrial profiles, were examined for each sample, and the ratios of mitochondria per cell profile, cytoplasmic membrane complexes per cell profile and mitochondrial inclusions per mitochondrial profile were calculated. The data were evaluated by use of standard deviation and Student's $t$ test for significant deviation, carried out at a two-sided rejection rate of 0.025 .

Under certain conditions, glutaraldehyde fixation has been reported to cause artefacts in the form of myelin-like whorls (see Discussion). Therefore, cells were fixed also with $\mathrm{KMnO}_{4}$ or cryofixed and examined as freeze-fractured preparations. Permanganate fixation was carried out at $0{ }^{\circ} \mathrm{C}$ with $\mathrm{I} \%(\mathrm{w} / \mathrm{v})$ aqueous $\mathrm{KMnO}_{4}$ for $2 \mathrm{~h}$. The sedimented cells were washed with $2 \%(\mathrm{w} / \mathrm{v})$ uranyl acetate before dehydration and embedded as described above. Freeze-etching was carried out by the general procedures outlined by Moor \& Muhlethaler (1963). Neurospora cells (non-glycerinated) were frozen on a platinum-gold specimen holder in Freon 22 at $-150^{\circ} \mathrm{C}$. Samples were fractured in a Balzers model 360 freezeetching unit. After fracturing and sometimes etching, samples were shadowed with platinum and carbon. Replicas obtained after digestion were transferred to grids and examined in a Siemens Elmiskop r.

Osmolality was measured on an Advanced Research Osmometer.

Reagents. Sucrose and glucose were Baker 'analysed' reagents. Mannitol, containing less than $250 \mu \mathrm{mol}$ reducing sugar/mol, was purchased from Mann Research Laboratories. Bacto-casitone and Bacto-yeast extract were obtained from Difco.

Lipid extraction. This was carried out on whole cells after $3 \mathrm{~h}$ growth in Vogel's medium with $15 \%(w / v)$ glucose as the carbon source. Cells were harvested and suspended in $3 \%(\mathrm{v} / \mathrm{v})$ glutaraldehyde $\mathrm{pH} 7.4$ for $2 \mathrm{~h}$ before extraction. The fixed cells were washed in $0.25 \mathrm{M}$-sucrose and suspended in cold chloroform-methanol ( 2 : i by vol.) or cold $90 \%(\mathrm{v} / \mathrm{v})$ aqueous acetone; the cells were stirred on ice for $2 \mathrm{~h}$. Following extraction, the samples were fixed with osmium tetroxide and embedded as described above.

\section{RESULTS}

The conversion of dormant conidiospores into rapidly growing hyphae may be measured morphologically by the appearance of the germ tube or gravimetrically by changes in the dry weight of the culture. Under the growth conditions employed here, germination, or appearance of the germ tube monitored by phase-contrast microscopy, increased linearly during the period between 2 and $3.5 \mathrm{~h}$ after inoculation (Fig. $\mathrm{I}$ ). After $5 \mathrm{~h}$ growth in $2 \%(w / v)$ sucrose, essentially all the cells in the culture had germinated. The rapidity with which the culture approached complete germination indicates that a high degree of synchrony of cellular activity existed.

The growth of Neurospora cells, as measured by dry mass, yielded a typical three-phase 


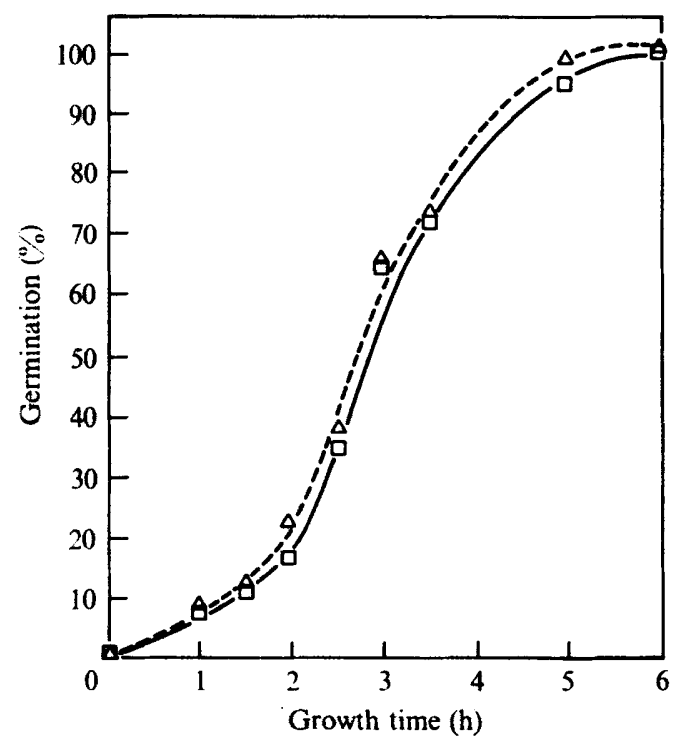

Fig. I

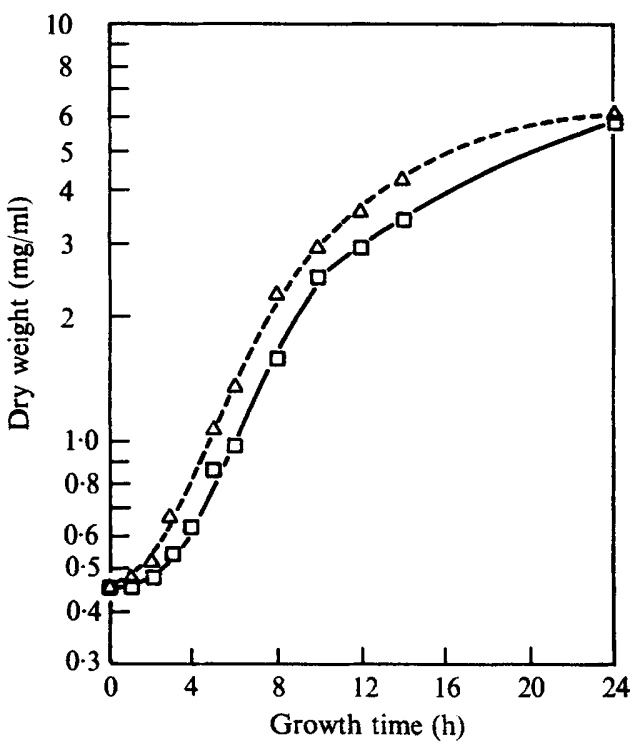

Fig. 2

Fig. I. Germination of Neurospora crassa. Samples of Neurospora were taken at various times during growth in medium containing $(\Delta) 2 \%$ sucrose or $(\square) 15 \%$ glucose. The fraction of cells showing any sign of germ tube extrusion was determined. Each time point represents an average of five experiments and a total of 1500 cells.

Fig. 2. Growth of Neurospora crassa. Cellular dry mass was measured gravimetrically at various times during growth in $(\triangle) 2 \%$ sucrose- or () $15 \%$ glucose-supplemented medium. Mass doubling time during exponential growth was $2.8 \mathrm{~h}$ in sucrose and $3.0 \mathrm{~h}$ in glucose.

growth curve (Fig. 2). In medium containing $2 \%$ sucrose, Neurospora cells exhibited a lag period of approximately $2 \mathrm{~h}$ duration, and then at about $3.5 \mathrm{~h}$ entered an exponential phase with a mass doubling time of $2.8 \mathrm{~h}$. The lag in germination and in growth (dry weight) were temporally related, in that entry of the culture into exponential growth was preceded by germination of 50 to $60 \%$ of the cells in the culture and germination was completed when cells were in early exponential phase of growth.

Experiments were designed to determine the effect of $15 \%(w / v)$ glucose, a carbohydrate concentration known to be highly repressive in yeast, on the growth and development of Neurospora cells. Although $15 \%$ glucose did not affect the germination of conidia (Fig. I), it gave a slightly prolonged lag phase (Fig. 2). Thus, in both media dedifferentiative events were initiated prior to $3.5 \mathrm{~h}$ and overlapped with the onset of redifferentiation which was completed by about $5 \mathrm{~h}$. In medium containing $15 \%(\mathrm{w} / \mathrm{v})$ mannitol, the growth curve had the same shape as those for glucose and sucrose except that the mass doubling time in the exponential portion was $4.3 \mathrm{~h}$.

Examination of the ultrastructure of a typical Neurospora cell showed that it contained one to several nuclei (each with a nucleolar region), a number of mitochondria, a cytoplasm densely packed with ribosomes, extremely small amounts of smooth membrane similar in appearance to the endoplasmic reticulum of mammalian cells (Bruni \& Porter, 1965), and a vacuole(s) extending throughout the cytoplasm (Fig. 3). The mitochondria of conidia are approximately $0.5 \mu \mathrm{m}$ in diameter and contain numerous cristae; in older cells the mitochondria are somewhat larger and more irregular in shape. However, the mitochondria in conidia retain essentially the same ultrastructural features of mitochondria in vegetative 


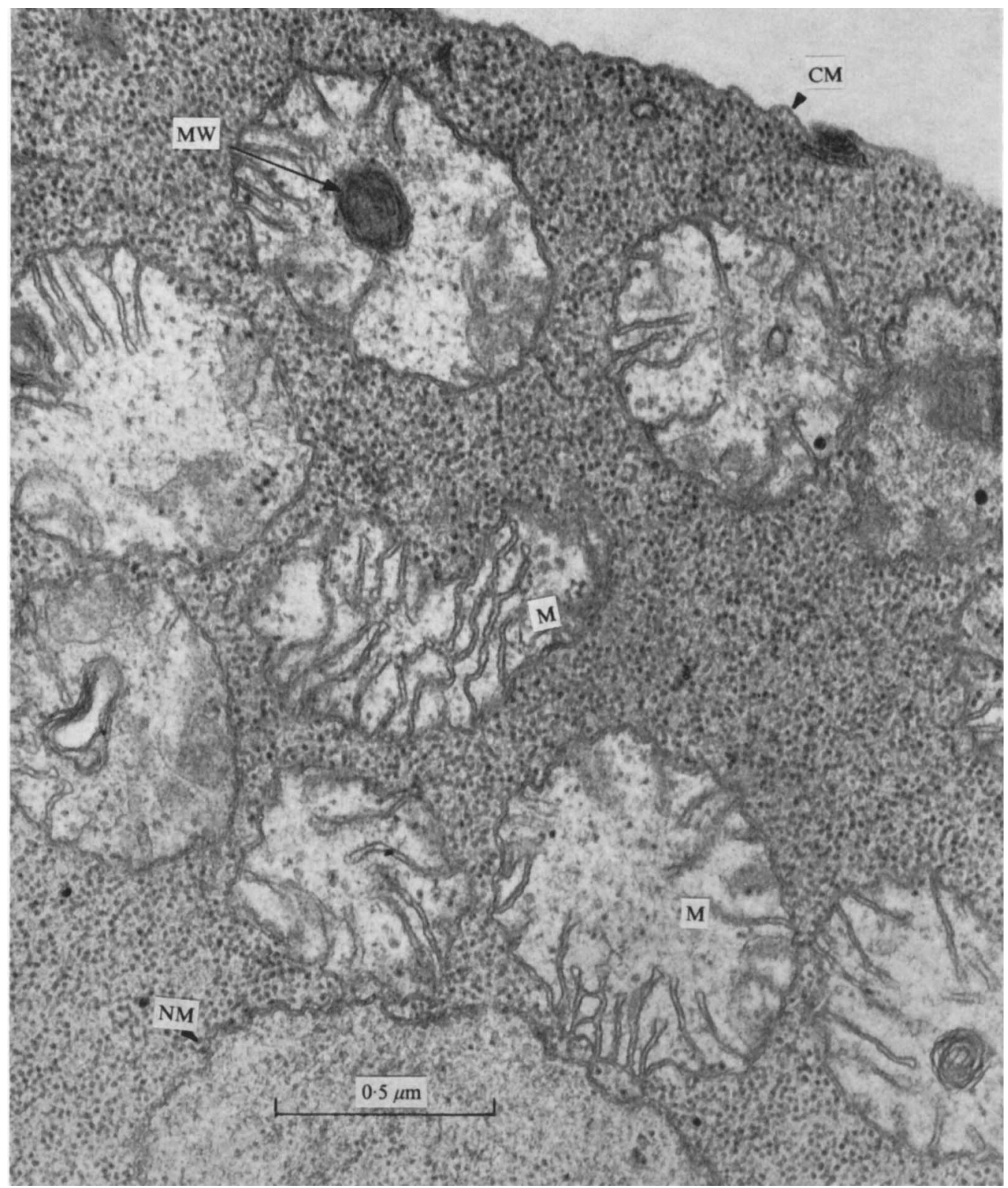

Fig. 3. Ultrastructure of Neurospora cell grown for $3 \mathrm{~h}$ in $2 \%$ sucrose. In this overview, the basic cytological features of Neurospora crassa are visible. The plasma membrane, mitochondrial inclusions, the high ribosome content, and a portion of a nucleus are included. Abbreviations used throughout the electron micrographs are as follows: A, hyaline region in mitochondrion; CM, cytoplasmic membrane; $\mathrm{CW}$, cytoplasmic whorl; F, filamentous material, DNA-like; $\mathrm{M}$, mitochondrion; MW, mitochondrial whorl; N, nucleus; NL, nucleolus; NM, nuclear membrane. 


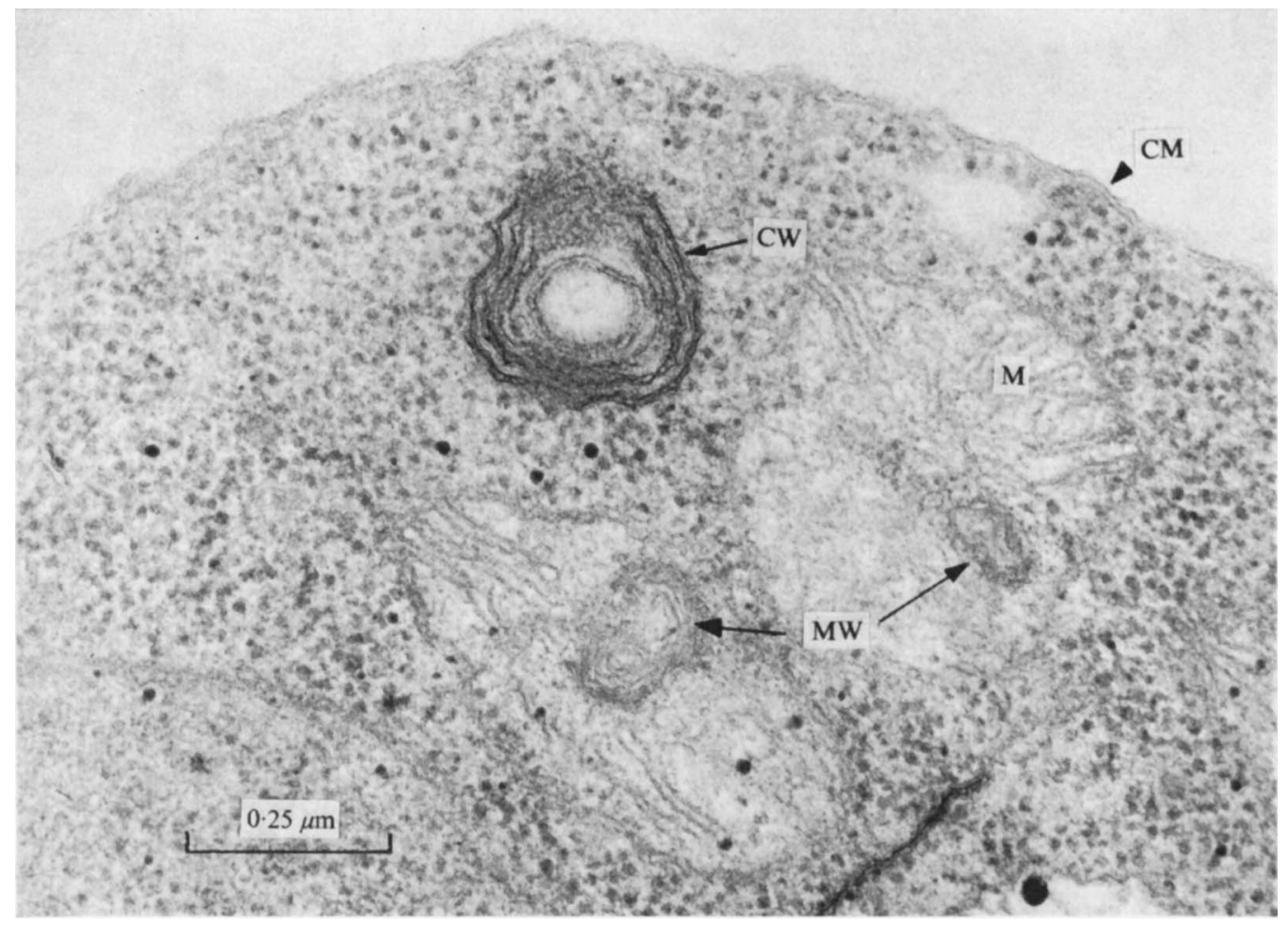

Fig. 4. Comparison of mitochondrial and cytoplasmic whorls, demonstrating the differences between the ultrastructural appearances of the cytoplasmic and mitochondrial membranous inclusions. For abbreviations, see Fig. 3.

cells. Thus, the differentiation of hyphae into dormant spores with the loss of mitochondrial enzymic activities (Turian, 1966; Stine, 1967) is not reflected in structural changes detectable at the electron microscope level. The cytoplasmic membrane of Neurospora cells was conspicuously asymmetric in its staining properties (see inset, Fig. $5 b$ ).

The cytology of Neurospora varied somewhat during growth. Whorled inclusions were observed in the mitochondria at specific times during growth, in agreement with Greenawalt (1965) and Beck \& Greenawalt (1968). These structures were composed of irregular rings of intermediate electron density which were slightly thinner than a 'unit membrane' (Figs. 3, 4). The material composing these structures was osmiophilic and could be seen in glutaraldehyde-osmium-fixed cells which had been stained by uranyl or lead salts. These mitochondrial inclusions differed from classical myelin-like whorls in that they did not appear to contain a continuous whorl of concentrically arranged, uniformly spaced (trilamellar), membranous material. Their shapes varied considerably from round to highly convoluted, with an average diameter of approximately $0.1 \mu \mathrm{m}$. Extensive examination of serial sections showed that the whorls lay in the mitochondrial matrix without any apparent continuity with the cristal membranes. Serial sections also showed that several separate inclusions might be present in a single mitochondrion.

We also observed membranous whorls in the cytoplasm of Neurospora cells (Fig. 4). They may be distinguished ultrastructurally from the mitochondrial whorls by two criteria. First, cytoplasmic whorls often occur in close promixity to and, at least in some 

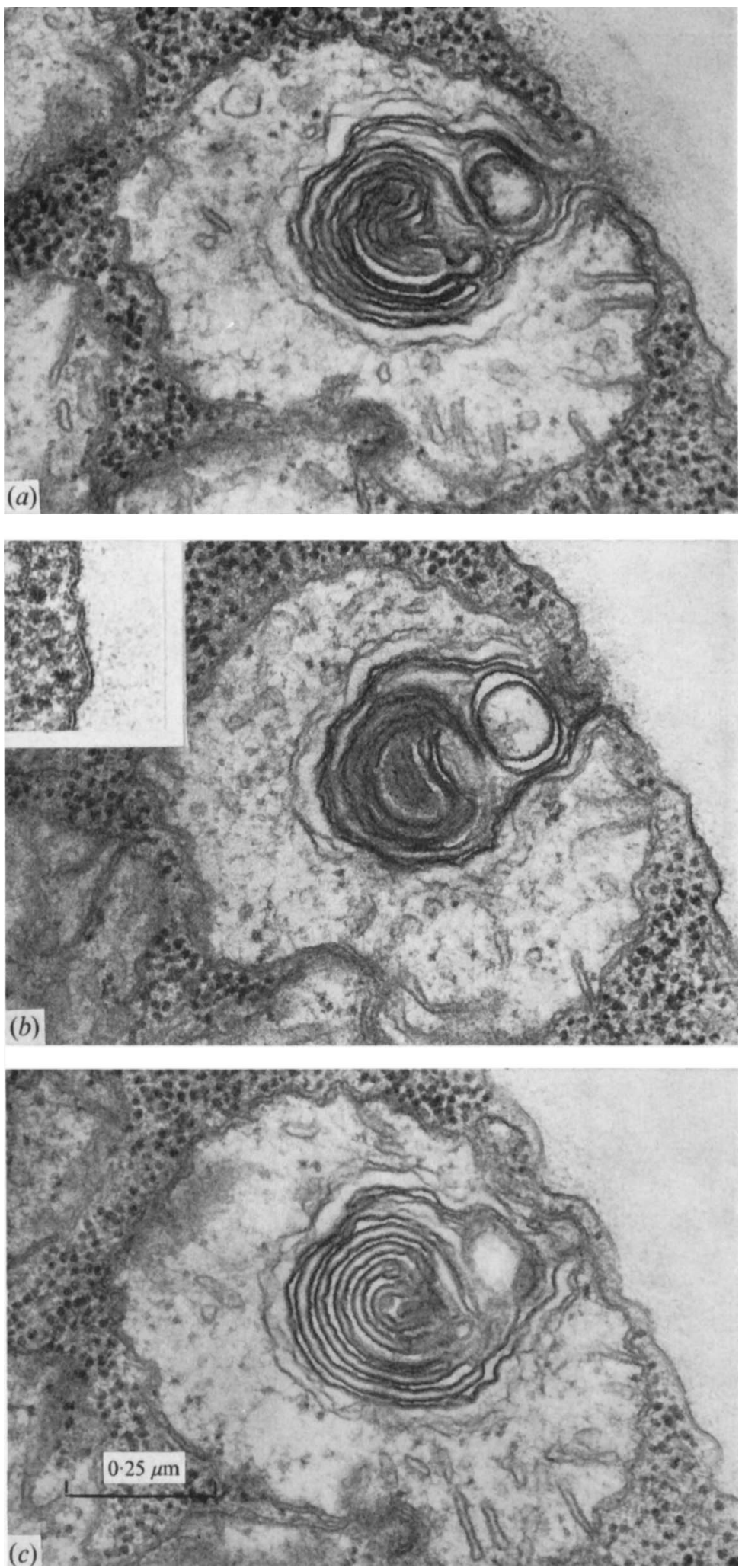

Fig. 5. Serial section through a cytoplasmic membrane whorl, showing its association with the cytoplasmic membrane. The continuity is most clearly shown in $(b)$. The asymmetry in electron density of the cytoplasmic membrane whorl is apparent. For comparison, an inset showing the asymmetry of the cytoplasmic membrane at $\mathrm{I} \cdot 2$ times higher magnification is included in $(b)$. A mitochondrion is seen in close proximity to this cytoplasmic whorl. 


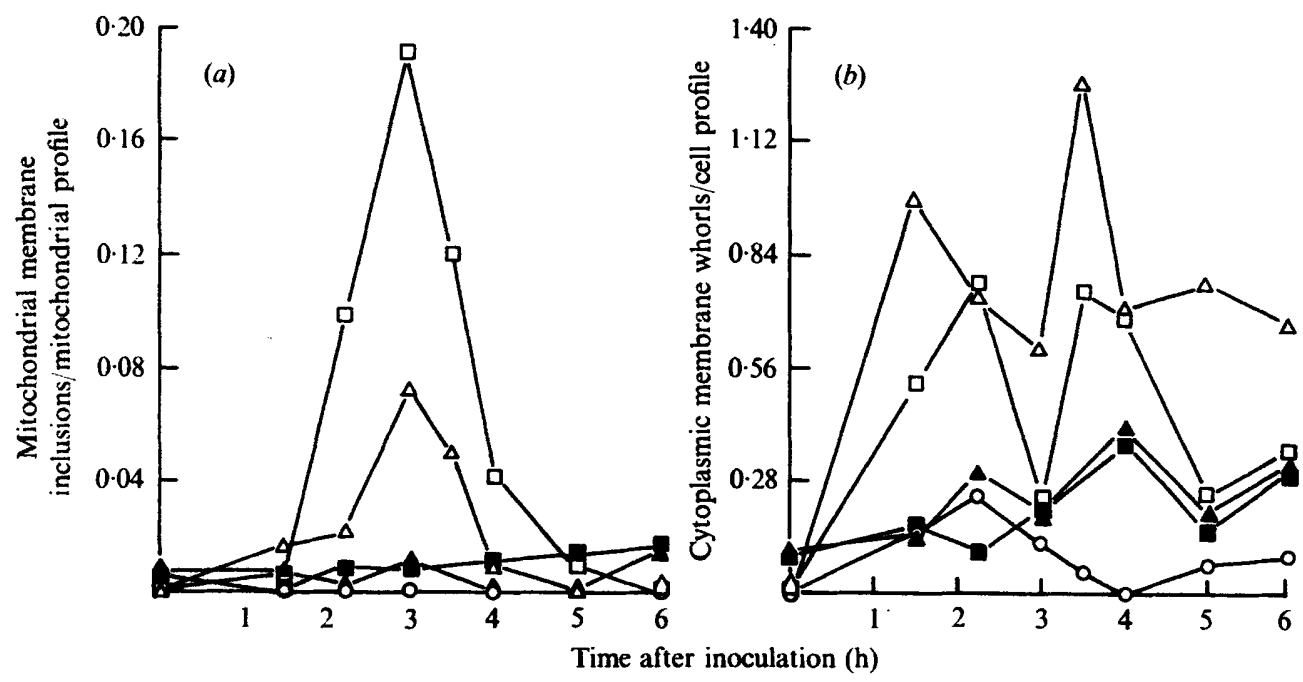

Fig. 6. Carbohydrate- and time-dependence of mitochondrial and cytoplasmic whorl formation. (a) The number of mitochondrial inclusions in cells grown in $(\triangle) 2 \%$ sucrose, ( $\square$ ) $15 \%$ glucose, (i) $15 \%$ mannitol, $(O) 13 \%$ 2-deoxyglucose, or $(\Delta)$ in the absence of added carbohydrate was counted. Values plotted for each time point represent observation of 30 to 40 cell profiles, each containing 8 to 10 mitochondria. (b) The number of cytoplasmic whorls in cells grown in $(\Delta)$ $2 \%$ sucrose, ( $\square$ ) $15 \%$ glucose, (D) $15 \%$ mannitol, (O) $13 \% 2$-deoxyglucose, or $(\Delta)$ in the absence of added carbohydrate. Values plotted represent the averages obtained from 30 to 40 cells at each time point.

cases, are continuous with the cytoplasmic membrane; second, these membranes are markedly different in appearance from the mitochondrial whorls. The cytoplasmic whorl often is composed of an asymmetrically staining membrane (see inset, Fig. $5 b$ ) which may be seen in transverse section to be wound around itself in a manner similar to the membranes of myelin. These characteristics are illustrated in three serial sections in Fig. 5, which also show the continuity of the cytoplasmic membrane and the cytoplasmic whorls. In Fig. 5, there is a mitochondrion in close association with, but clearly separate from, a cytoplasmic whorl. Such an association was very infrequently observed in our studies. It seems likely, however, that this type of association in part led Malhotra (I968) to postulate the existence of mesosomes in Neurospora.

That the occurrence of membranous inclusions in $N$. crassa mitochondria was both time-dependent and carbohydrate-dependent was investigated further by electron microscopy of Neurospora cells during the first $6 \mathrm{~h}$ of growth. The number of profiles of mitochondria, mitochondrial whorls, and cytoplasmic whorls were counted and the results were analysed statistically as described in Methods. The number of mitochondrial whorls per mitochondrial profile reached a peak sharply at $3 \mathrm{~h}$ growth in both sucroseand glucose-grown cells (Fig. 6a), and the number of mitochondrial whorls in glucosegrown cells was approximately three times greater than the number in sucrose-grown cells. These results were highly reproducible.

Why there are more mitochondrial inclusions in the glucose-grown than in the sucrosegrown cells is not known. We considered two of the possible causes: an osmotic effect brought about by the high osmolality of the glucose-supplemented medium, and a metabolic effect related, perhaps, to glucose utilization and repression of mitochondrial function (known to occur in yeast cells). 
To test the first possibility, cells were grown in a medium containing $2 \%(w / v)$ sucrose plus $13 \%(w / v)$ 2-deoxyglucose and examined for the appearance of membrane whorls in mitochondria. In Neurospora, 2-deoxyglucose is transported but not phosphorylated (Neville, Suskind \& Roseman, 1971), and competitively inhibits both glucose and fructose transport (Marzluff \& Metzenberg, 1967); it is likely that 2-deoxyglucose also competes with glucose generated from internal trehalose (Bianchi \& Turian, 1967; Hanks \& Sussman, 1969) for sites on hexokinase. The high osmolality of the medium containing $13 \%$ 2-deoxyglucose and $2 \%$ sucrose simulates the osmolality of the growth medium containing $15 \%$ glucose. No mitochondrial whorls were seen in cells grown under these conditions (Fig. 6a).

To test whether the appearance of mitochondrial whorls in $2 \%(w / v)$ sucrose-supplemented medium and the elevated whorl production in $15 \%$ glucose-supplemented medium were due to the levels of glucose available to the cells [glucose is available from sucrose due to a high invertase activity in Neurospora (Trevithick \& Metzenberg, 1966)], cells were grown in $15 \%$ mannitol and examined for the production of mitochondrial whorls. Neurospora is able to use mannitol only slowly (Perlman, 1950) and exhibits a somewhat longer mass doubling time than when it is grown in sucrose or glucose. Mannitol thus provides both a growth-limiting carbohydrate source and, at the level of $15 \%$, an osmotic effect as well. In this growth condition, only very few mitochondrial whorls were observed (Fig. $6 a$ ). In addition, cells germinated in the absence of carbohydrate supplementation contained very few mitochondrial whorls. Since supplementation with mannitol, deoxyglucose or the absence of added carbohydrate leads to prolonged mass doubling times, cells grown under these different conditions were examined for whorls when comparable dry weights had been reached. Few mitochondrial inclusions could be seen in these samples. The results of these experiments suggest that mitochondrial whorls are observed only in cells exposed to relatively high levels of metabolizable carbohydrate. Furthermore, since very few whorls are formed in the presence of high concentrations of mannitol or 2-deoxyglucose, the whorls are not produced non-specifically in response to the high osmolality of growth media.

The number of cytoplasmic whorls per cell profile also is dependent on the carbohydrate supplementation (Fig. 6b). In contrast to mitochondrial whorls, however, cytoplasmic whorls are seen in cells under growth-limiting conditions. The level of formation of cytoplasmic whorls in mannitol is about $25 \%$ of that found in sucrose- and glucose-grown cells, and whorls are formed at a low but finite level in $13 \%(w / v) 2$-deoxyglucose. Also, in contrast to the mitochondrial structures, the temporal appearance of the cytoplasmic whorls is variable in sucrose- and glucose-grown cultures.

The effects of several lipid solvents on the ultrastructure of Neurospora were studied, since the osmiophilic nature of the mitochondrial whorls indicated that they might be composed partly of lipid. If the whorls contain lipid, they might be expected to be more soluble in a lipid solvent than are the lipids which are fully integrated into the membrane. For example, the data of Ashworth et al. (1966) show that the lipids of hepatic osmiophilic droplets exhibit a greater solubility in lipid solvents than do the membrane lipids.

Extraction of Neurospora cells with cold $90 \%$ aqueous acetone followed by preparation for electron microscopy results in complete destruction of all cellular features. If, however, cells containing maximal numbers of mitochondrial whorls were first fixed with $3 \%$ glutaraldehyde for $\mathbf{2} \mathrm{h}$ and then extracted, the fundamental cellular organization remained intact (Fig. 7). Only limited extraction of the cytoplasmic membrane and cytoplasmic whorls occurred and the electron opacity and definition of the mitochondrial and nuclear 


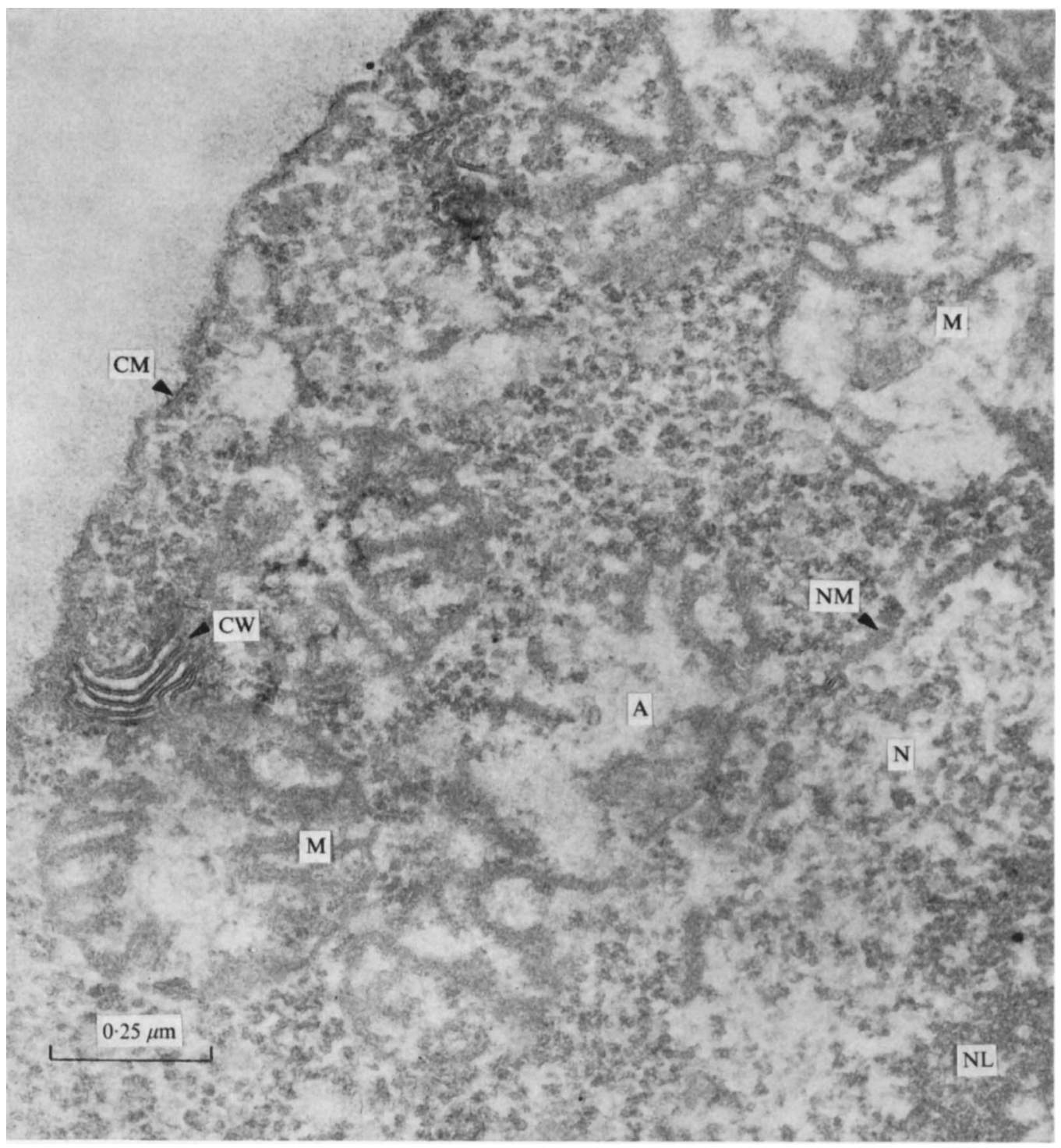

Fig. 7. Acetone-extracted Neurospora cell. Neurospora were grown for 3 h in $15 \%$ glucose, fixed for $2 \mathrm{~h}$ with $3 \%$ glutaraldehyde and then extracted for $2 \mathrm{~h}$ with cold $90 \%$ aqueous acetone. There are no morphologically-identifiable mitochondrial inclusions, but within the mitochondria are hyaline regions containing filamentous material. Other cellular membranes, including cytoplasmic whorls, remain largely intact. For abbreviations, see Fig. 3.

membranes were reduced. Mitochondrial whorls, however, were removed completely and could not be seen even after exhaustive examination of many mitochondria. The extracted mitochondria were marked by hyaline areas (A) within which were fine filaments (Figs. 7, 8). Extraction of prefixed cells with chloroform-methanol (2:I) gave similar results; however, the general cellular organization and ultrastructure were less well preserved and the intramitochondrial filaments were grossly clumped.

The fact that glutaraldehyde fixation of cells can result in the formation of myelin-like 


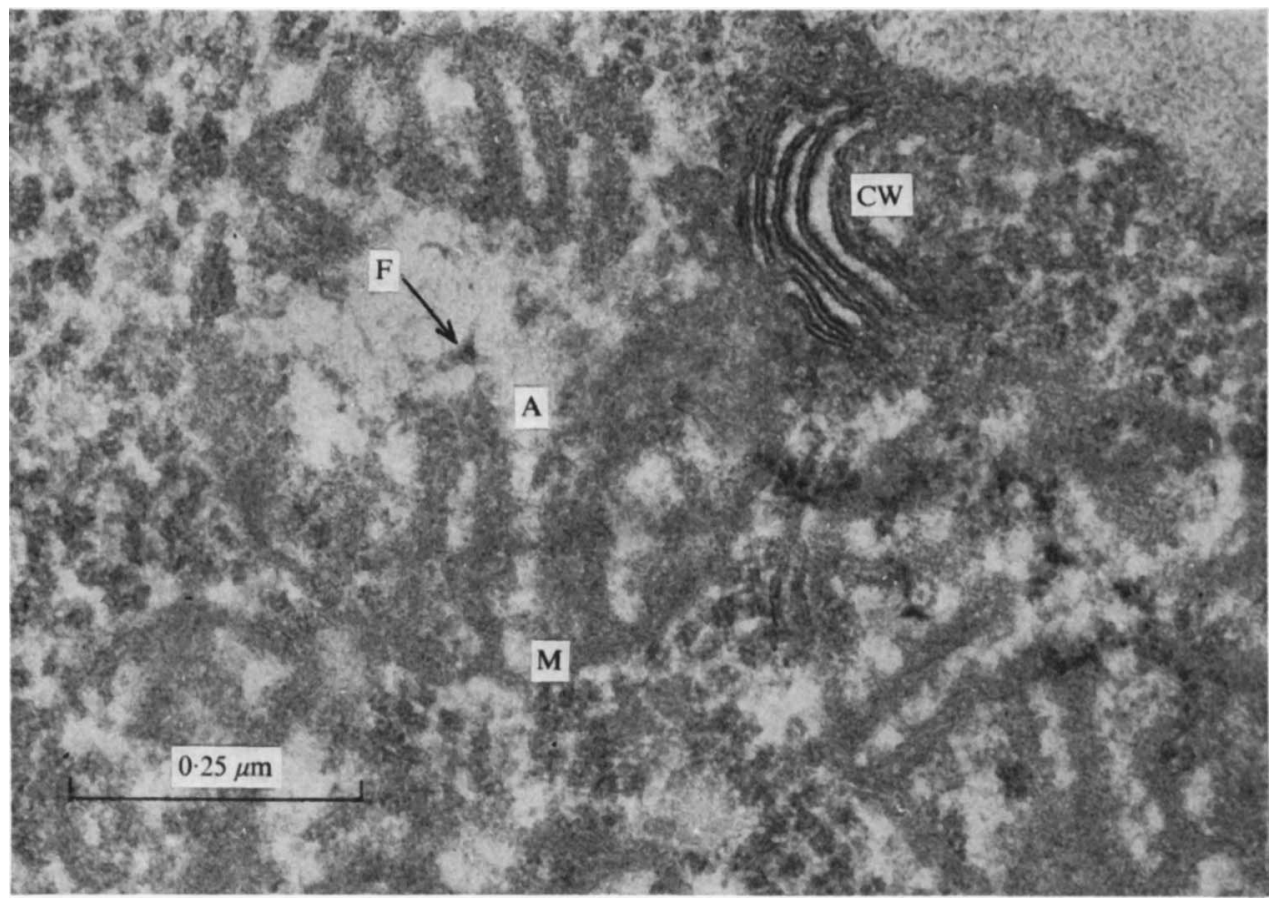

Fig. 8. Acetone-extracted cell at high magnification. Only the general mitochondrial morphology is retained but the cytoplasmic whorl remains largely intact and well defined, and the DNA-like filaments are clearly seen. For abbreviations, see Fig. 3.

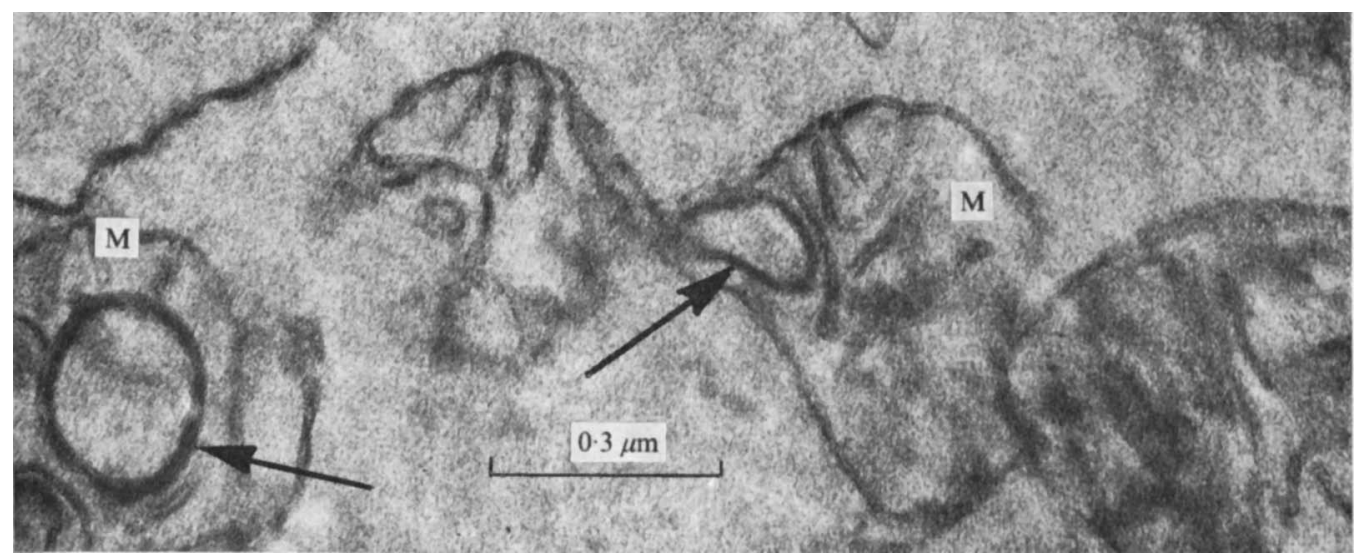

Fig. 9. Membranous whorls in mitochondria of permanganate-fixed cells. Cells grown for $3.5 \mathrm{~h}$ in $15 \%$ glucose were fixed with $1 \% \mathrm{KMnO}_{4}$ and washed with uranyl acetate as described in Methods. Three distinct myelin-like figures can be seen in different mitochondria (arrows). $M$, mitochondrion. 


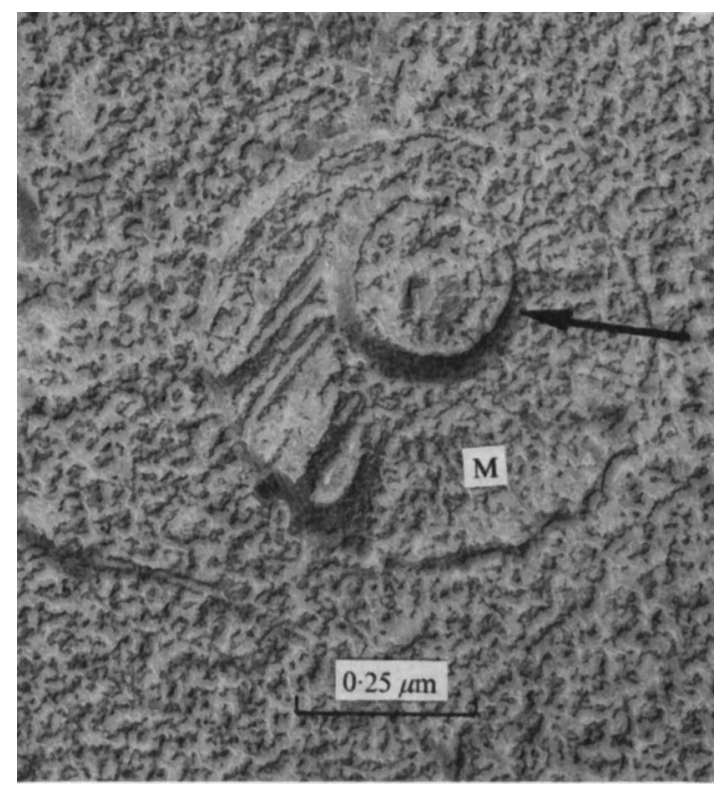

Fig. 10. Mitochondrial inclusions in freeze-fractured cells. Neurospora were grown for $3 \mathrm{~h}$ in $15 \%$ glucose. The cells were frozen immediately at $-150{ }^{\circ} \mathrm{C}$ in Freon 22 , then fractured and etched as described in Methods. Several mitochondria are shown, one of which contains an example of the intramitochondrial structures observed in freeze-etched preparations. $M$, mitochondrion.

figures under certain conditions (Curgy, 1968) prompted the use of other fixation procedures to investigate the structural reality of these inclusions. Mitochondria in cells grown in $15 \%$ glucose medium to provide maximal whorl production and then fixed with permanganate did exhibit profiles of membrane whorls (Fig. 9). Presumably, these images represent the same structures seen routinely in glutaraldehyde-osmium-fixed cells but the gross differences in appearance of the cells fixed with $\mathrm{KMnO}_{4}$, and the well-known destructive effects of this fixative, do not permit a final conclusion in this regard. Therefore, glutaraldehyde-fixed and unfixed cells shown by thin sectioning to contain large numbers of mitochondrial whorls were cryofixed for freeze-fracturing (Moor \& Muhlethaler, 1963). Mitochondria in fixed cells appeared occasionally to contain inclusions (see Fig. 10), possibly the whorls seen in thin sections. However, analysis was complicated by the fact that fracturing in fixed cells seemed to occur more frequently over or under the mitochondrion rather than across the mitochondrial membranes and through the matrix.

\section{DISCUSSION}

The initiation of dedifferentiative and redifferentiative events in Neurospora crassa reflects the activation and regulation of cellular metabolism. We have carried out a detailed electron microscopic examination of germinating conidiospores to monitor ultrastructural changes accompanying these metabolic alterations. Although the basic ultrastructural features of Neurospora cells remained relatively constant during germination and exponential growth, several unusual structures were observed at certain times. The whorled structures which appeared in the mitochondria are of particular interest in our search for morphological correlates of biochemical differentiation. Cochrane (1966) and others 
(Turian, 1966; Stine, 1967) have shown that a shift from fermentative to oxidative metabolism occurs during the germination period. We have found that crude cell homogenates of hyphae had a 6- to ro-fold greater specific activity of succinic dehydrogenase than similar preparations of conidia and that a 3 -fold greater specific activity of lactic dehydrogenase was present in conidia than in hyphae (unpublished observation). Thus, it might reasonably be expected that the biochemical changes observed are related to the development of mitochondrial functions, which in turn are dependent upon the synthesis and/or assembly of mitochondrial membranes.

The membranous mitochondrial whorls were osmiophilic and could be removed from the cells by treatment with $90 \%$ aqueous acetone in the cold; both of these characteristics indicate that they are composed at least partly of lipid. We have considered the possibility that these structures may be analogous to bacterial mesosomes as suggested by Malhotra (I968). Mesosomes isolated from Staphylococcus aureus have a high lipid content relative to the plasma membrane (Theodore \& Panos, 1973). However, such considerations, in our opinion, are entirely speculative at the moment and, probably, premature since the formation, structure, and function of bacterial mesosomes remain unclear.

The strong dependence of the number of mitochondrial whorls on time and carbohydratesupplementation indicates that there is a physiological basis for the presence of these structures. However, it has been reported that slow penetration of glutaraldehyde into tissues can result in the formation of artefacts which appear as whorled myelin-like membranes (Ericsson \& Biberfeld, 1967). In our experiments, Neurospora cells were fixed in suspension. Under these conditions, cellular respiration measured polarographically was inhibited completely within I min of glutaraldehyde being added to a final concentration of $3 \%$, which indicates that glutaraldehyde penetrates rapidly into the mitochondria. Curgy (1968) suggested that mobilization of cellular membrane lipids may occur during glutaraldehyde fixation and that, during subsequent osmium fixation, the lipid is fixed in association with existing membrane with concomitant myelinization. In this regard, serial sections analysed in the present study showed no obvious association of the mitochondrial whorls with cristal membranes. Furthermore, the fact that formation of the whorls in Neurospora mitochondria is time- and carbohydrate-dependent strongly indicates that even if the images seen do not represent precisely the native state of these mitochondria, they nevertheless do reflect changes occurring in the mitochondria at times significant to cellular differentiation before the onset of rapid cell growth.

The synthesis and content of lipids, particularly of mitochondrial lipids, might therefore be high during the period of maximal whorl formation, and the high concentration of glucose effecting mitochondrial whorl formation might be reflected in the rate of lipid synthesis. The accompanying paper (Beck \& Greenawalt, 1976) describes and correlates the synthesis of mitochondrial lipids with the development of mitochondrial function and the presence of membranous inclusions during germination and growth of Neurospora crassa conidia.

This research was supported by research grants GM12125 from the National Institutes of Health, GB31098 from the National Science Foundation, and by USPHS Graduate Training Grant GMoor 84 to the Department of Physiological Chemistry, Johns Hopkins University School of Medicine. We are indebted to Dr Jeanne C. Beck for her generous help in reading and preparing the manuscript and to Mr G. L. Decker and Mr R. A. Garrett for expert technical assistance. We thank Dr Charles Hackenbrock for the freezeetched preparation. 


\section{REFERENCES}

Ashworth, C., Leonard, J., Eigenbrodt, E. \& Wrightsman, F. (1966). Hepatic intracellular osmiophillic droplets. Effect of lipid solvents during tissue preparation. Journal of Cell Biology 31, 301-318.

Beck, D. P. \& Greenawalt, J. W. (1968). Factors affecting the formation of membranous structures in the cytoplasm and mitochondria of Neurospora crassa. Journal of Cell Biology 39, I A.

BeCK, D. P. \& GreenawALT, J. W. (1976). Biogenesis of mitochondrial membranes in Neurospora crassa during cellular differentiation. II. Changes in oxidative phosphorylation and synthesis of mitochondrial phospholipids. Journal of General Microbiology 92, I I-19.

BiANCH, D. \& TURIAN, G. (1967). Lipid content of conidia of Neurospora crassa. Nature, London 214, I344-1345.

Bruni, C. \& PORTER, K. (1965). The fine structure of the parenchymal cells of the normal rat liver. I. General observations. American Journal of Pathology 46, 691-755.

Cochrane, V. W. (1966). Respiration and spore germination. In The Fungus Spore, pp. 20I-2I5. Edited by M. F. Madelin. London: Butterworths.

Curgy, J.-J. (1968). Influence du mcde de fixation sur la possibilité d'observer des structures myeliniques dans les hépatocytes d'embryons de poulet. Journal de Microscopie 7, 63-80.

Ericsson, J. \& Biberfeld, P. (1967). Studies on aldehyde fixation: fixation rates and their relation to fine structure and some histochemical relations in liver. Laboratory Investigation 7, 281-298.

GreenaWALT, J. W. (1965). The effect of growth conditions on the cytology of Neurospora crassa. Journal of Cell Biology 27, 37 A.

Hall, D. O. \& Greenawalt, J. W. (I964). Oxidative phosphorylation by isolated mitochondria of Neurospora crassa. Biochemical and Biophysical Research Communications 17, 565-569.

Hall, D. O. \& Greenawalt, J. W. (1967). The preparation and biochemical properties of mitochondria from Neurospora crassa. Journal of General Microbiology 48, 419-430.

HaNks, D. \& Sussman, A. (1969). The relation between growth, conidiation and trehalase activity in Neurospora crassa. American Journal of Botany 56, $1152-1159$.

LUFT, J. H. (1961). Improvements in epoxy resin embedding methods. Journal of Biophysical and Biochemical Cytology 9, 409-4I4.

Malhotra, S. K. (I968). Mesosome-like structures of poky Neurospora. Nature, London 219, 1267-1 268.

Marzluf, G. \& Metzenberg, R. (1967). Studies on the functional significance of the transmembrane location of invertase in Neurospora crassa. Archives of Biochemistry and Biophysics 120, 487-496.

Minlonig, G. (1961). Advantages of a phosphate buffer for $\mathrm{OsO}_{4}$ solutions in fixation. Journal of Applied Physiology 32, 1637-1639.

Millonig, G. (1962). Further observations on a phosphate buffer for osmium solutions. In Fifth International Congress for Electron Microscopy, vol. 2, p. 8. Edited by S. S. Breese. New York: Academic Press.

Moor, H. \& Muhlethaler, K. (1963). Fine structure in frozen-etched yeast cells. Journal of Cell Biology $17,609-628$.

Neville, M. Suskind, S. \& Roseman, S. (1971). A derepressible active transport system for glucose in Neurospora crassa. Journal of Biological Chemistry 246, $1294-1301$.

Perlman, D. (1950). The metabolism of carbohydrates by Neurospora. Bulletin of the Torrey Botanical Club 77, 103-109.

ReYNOLDS, E. S. (1963). The use of lead citrate at high pH as an electron-opaque stain in electron microscopy. Journal of Cell Biology 17, 208-212.

Sabatini, D. D., Bensch, K. \& Barnett, R. J. (1963). Cytochemistry and electron microscopy. The preservation of cellular ultrastructure and enzymatic activity by aldehyde fixation. Journal of Cell Biology 17, 19-58.

StiNe, G. J. (1967). Enzyme activities during the asexual cycle of Neurospora crassa. I. Succinic dehydrogenase. Canadian Journal of Microbiology 13, I 203-1 210.

STINE, G. J. (1969). Investigations during phases of synchronous development and differentiation in Neurospora crassa. In The Cell Cycle, pp. I19-139. Edited by G. M. Padilla, G. L. Whitson and I. L. Cameron. New York: Academic Press.

Theodore, T.S. \& Panos, C. (1973). Protein and fatty acid composition of mesosomal vesicles and plasma membranes of Staphylococcus aureus. Journal of Bacteriology 116, 57 I-576.

Trevithick, J. \& METZENBERG, R. (1966). Molecular sieving by Neurospora cell walls during secretion of invertase isozymes. Journal of Bacteriology 92, 1010-1015.

TURIAN, G. (1966). Morphogenesis in ascomycetes. In The Fungi: An Advanced Treatise. II. The Fungal Organism, pp. 339-385. Edited by G. C. Ainsworth and A. S. Sussman. New York: Academic Press.

Vogel, H. J. (1956). A convenient growth medium for Neurospora (medium N). Microbiology and Genetics Bulletin 13, 42-43.

WEISS, B. (1965). An electron microscope and biochemical study of Neurospora crassa during development. Journal of General Microbiology 39, 85-94.

ZaloKAR, M. (1959). Enzyme activity and cell differentiation in Neurospora. American Journal of Botany 46, 555-559. 